\title{
Evaluación de la investigación cubana en Comunicación Social: ¿reto o necesidad?
}

\author{
Assessment of Cuban research in Social \\ Communication: Challenge or necessity?
}

\author{
Yelina PIEDRA-SALOMÓN' \\ Dasniel OLIVERA-PÉREZ ${ }^{2}$ \\ Víctor HERRERO-SOLANA ${ }^{3}$
}

\section{Resumen}

Desde una perspectiva teórica se expone la importancia y necesidad de desarrollar estrategias sistemáticas de evaluación de la investigación en Comunicación Social en Cuba para perfeccionar su gestión. Se aborda brevemente la evaluación de la ciencia haciendo énfasis en las Ciencias Sociales, sirviéndose de las potencialidades de las herramientas métricas para realizar ejercicios evaluativos sistemáticos y profundos. Se alude a las condicionantes y características de la investigación del campo en cuestión dado su "carácter profesionalizante", el escaso valor otorgado a la investigación teórico-histórica, la incipiente existencia de publicaciones nacionales, la nula visibilidad internacional de la producción científica nacional a partir de fuentes propias además de la inexistencia de un modelo de gestión de la investigación y de un sistema de información científica. Se identifican potencialidades para diseñar una sólida batería de indicadores en consonancia con la realidad del dominio en cuestión y la consecución de la evaluación como eje central de una política científica nacional que incentive la investigación en comunicación, bien como su gestión y desarrollo.

Palabras clave: Comunicación social. Cuba. Estudios bibliométricos. Evaluación científica. Investigación científica. Política de investigación.

\begin{abstract}
From a theoretical perspective the present contribution concentrates on exposing the importance and necessity of developing systematic strategies of research evaluation to improve management in the Cuban communication field. The evaluation of the topic is briefly addressed by emphasizing Social Sciences. This paper also presents the potentialities of the metric tools which carry out intensive and systematic evaluation exercises. Conditions and characteristic of the research in the field are presented in order to explain its "professional character", scarce value given to the theoretical-historical research, incipient existence of national publications, null international visibility of the national scientific production relying on its own sources, nonexistence of a model of research management and of a scientific information system. Potentialities are identified in order to elaborate a solid group of indicators in total harmony with the reality and characteristic of the issue in question, as well as to conceive the evaluation as the central axis of a national scientific politics that motivates research in communication, its management and development.
\end{abstract}

Keywords: Social communication. Cuba. Bibliometric studies. Scientific evaluation. Scientific research. Research policy.

\footnotetext{
1 Universidad de La Habana, Facultad de Comunicación, Departamento Ciencias de la Información. Av. Independencia esq. San Pedro, Edificio Bohemia, Código-Postal 10400, La Habana, Cuba. Correspondencia a nombre de/Correspondenceto:Y.PIEDRA-SALOMÓN. E-mail: <yelinapiedra@fcom.uh.cu>.

2 Universidad de La Habana, Facultad de Comunicación, Departamento Comunicación Social. La Habana, Cuba.

3 Universidad de Granada, Facultad de Información y Comunicación, Departamento Documentación. Granada, España.

Recebido el 19/1/2015 y aceptado para su publicación el 21/7/2015.
} 


\section{Introducción}

Existe amplio consenso sobre la importancia central de la ciencia y la tecnología para el desarrollo de las sociedades contemporáneas. En este sentido, la literatura disponible refleja esta apreciación al mostrar los efectos generales del conocimiento en el crecimiento económico así como los efectos directos o indirectos que a su vez pueden tener la ciencia y la tecnología sobre el desarrollo social.

En nuestros días se hace cada vez más evidente la aplicación de métodos y procedimientos matemáticos para la evaluación de la actividad científica como mecanismo operativo e instrumento de las políticas nacionales de ciencia. La aplicación de métodos basados en el uso de los indicadores métricos se sustenta principalmente en el análisis de bases de datos bibliográficas que permiten la cuantificación de grandes volúmenes de publicaciones científicas en cualquier área de conocimiento.

La Bibliometría/Cienciometría, sin hacer énfasis en distinciones conceptuales, es uno de los campos de la investigación verdaderamente interdisciplinarios extendido a casi todos los campos científicos. Si bien la metría ha evolucionado satisfactoriamente hasta llegar a lo que hoy se conoce como Almetrics, es sensible de ser dividida en tres subgrupos que definen claramente los tópicos de la metría contemporánea: para bibliómetras, para las disciplinas científicas y para la gestión y evaluación de políticas científicas. Esta última se desarrolla a diferentes niveles, donde concretamente la evaluación de la investigación tiene su campo de acción.

Los análisis bibliométricos avanzados son una poderosa herramienta para evaluar de manera fiable, transparente y objetiva la influencia internacional de los trabajos científicos y descubrir con los mapas de la ciencia patrones en la estructura de los campos que permiten identificar interdisciplinariedad y flujos de conocimiento, entre otros aspectos (Van Raan, 2014). El diseño e implementación de herramientas avanzadas para el monitoreo de universidades, departamentos e institutos, el establecimiento de indicadores de revistas académicas y los rankings de universidades y su representación han traído consigo que los métodos bibliométricos hayan alcanzado un alto reconocimiento como instrumentos informativos y fiables en la práctica de la evaluación de la investigación.

Hablar de evaluación de la investigación en el siglo XXI implica una concepción integradora además de multidimensional, donde se complementan diferentes métodos, mecanismos y modelos. Si bien las disciplinas métricas de la información resultan de extraordinaria importancia para la toma de decisiones estratégicas insertados en las políticas de gestión de la actividad científica y tecnológica, los datos e informaciones que registren deben ser interpretados en contextos discursivos, culturales, institucionales y políticos de producción de conocimientos.

En Cuba, los estudios de este corte se han venido fomentando en los últimos años; si bien guiados por estrategias institucionales y como parte formativa de un claro proceso marcado política y estratégicamente por el gobierno, también como parte de un impulso esencialmente universitario que ha contado con el apoyo de entidades nacionales e internacionales. Un ejemplo de esto lo constituye el programa doctoral en Biblioteconomía y Documentación desarrollado entre las universidades de La Habana y Granada, España - 20072016. Este programa ha contribuido a impulsar, sistematizar y consolidar las competencias de un conjunto de especialistas dedicados a este tipo de estudios en el país. No obstante, se hace necesario elevar el rigor de los análisis cuantitativos de la producción científica nacional con vistas a analizar las principales características de producción, su grado de especialización en los diversos ámbitos temáticos, la dimensión estructural de los fenómenos disciplinarios e interdisciplinarios relacionados con los resultados que dan a conocer, su recepción por parte de la comunidad científica nacional e internacional y la percepción de esta visibilidad como elemento para juzgar la calidad de las investigaciones científicas. Se trata de rebasar la mera aproximación cuantitativa e incidir en los procesos de toma de decisiones estratégicas encaminados a impulsar, consolidar o perfeccionar la evaluación de la actividad científica realizada en el país (Arencibia, 2012).

Según este autor, desarrollar un instrumento cienciométrico para el análisis y evaluación de los diferentes aspectos que caracterizan a la ciencia nacional, 
en franca relación con su entorno regional y desde dimensiones cuantitativas, cualitativas, socioeconómicas y estructurales, es el reto que enfrentan hoy los profesionales de la información involucrados en procesos de toma de decisiones estratégicas en materia de Ciencia, Tecnología e Innovación. Implementar este instrumento de manera sistemática, convertirlo en herramienta analítica para el perfeccionamiento continuo de una Política Nacional de Ciencia y Tecnología, y utilizarlo como guía complementaria de los estudios teóricos y epistemológicos presentes y futuros en torno al surgimiento, desarrollo y perspectivas de la actividad científica en el país, constituyen los desafíos emergentes.

La información emanada de estos estudios tributa a una idónea toma de decisiones en pos de lograr una mejor distribución de recursos para la investigación en las instituciones, así como para el fomento de nuevas políticas científicas o para la consolidación y legitimización de los diferentes espacios de conocimiento. Su aplicación proporciona elementos que obliga a las comunidades científicas a realizar sólidas reflexiones teóricas y epistemológicas hacia el interior de dichos espacios de conocimiento.

La presencia de manera significativa de las Ciencias Sociales en los Centros de Educación Superior de nuestro país, así como la imperante necesidad de tomar en cuenta el perfeccionamiento de la gestión de la actividad de investigación socio-humanística, dan cuenta de la necesidad de la introducción de indicadores y procedimientos de evaluación cienciométricos.

La importancia creciente de las investigaciones sociales en el ámbito nacional a partir desu correspondencia con la naturaleza misma de nuestro sistema y con las transformaciones, contradicciones y exigencias actuales del desarrollo social y económico exigen contribuir al perfeccionamiento de la planificación estratégica de la actividad científico-tecnológica dentro del plan de transformaciones que ha venido proponiendo la dirección del país. Se hace imprescindible un conocimiento más completo de las características y potencialidades de la actividad investigativa en el campo objeto de análisis así como el nivel de relación existente entre la actividad investigativa que desempeñan y las necesidades y demandas sociales.
Sobre la base de lo planteado hasta el momento es que la presente contribución se propone como principal objetivo exponer, a partir de la investigación documental, los puntos de partida de la evaluación de la investigación en el campo científico de la Comunicación en Cuba, los elementos condicionantes y los retos de su aplicación desde una perspectiva cienciométrica.

\section{Evaluación de la investigación en Ciencias Sociales}

Los dominios del conocimiento científico y las políticas nacionales de investigación convergen a partir de la asunción de que el análisis y la evaluación de la información y el conocimiento resultantes de la actividad científica son elementos imprescindibles para todos los programas de investigación pública, tecnología y desarrollo que se implementan en una sociedad (Arencibia \& Moya-Anegón, 2008). Según esta línea de pensamiento, en términos de producción científica y publicación en revistas con visibilidad internacional, los países del mundo pueden ser clasificados en tres grandes grupos (Biomédico, Ciencias Básicas e Ingeniería y Agricultura) de acuerdo a su perfil temático. Estos grupos se reflejan en modelos de comportamiento que resumen las características de sus sistemas de conocimiento e innovación (Moya-Anegón \& Herrero-Solana, 2013).

La necesidad de evaluar los resultados alcanzados por las políticas públicas de ciencia y tecnología ha obligado a desarrollar herramientas metodológicas capaces de situar en los diferentes niveles de agregación a esos dominios. Por eso, se hace necesario utilizar fuentes de información científica e indicadores que permitan la contextualización mencionada.

Una agenda de indicadores sugiere una agenda de relevancia toda vez que identifica lo que es importante y desencadena las actuaciones que intentarán alcanzarlo (Núñez, 2010). Por este motivo, las herramientas de evaluación de la producción científica que se creen específicamente para evaluar el producto de la ciencia deben ser elaboradas e investigadas de acuerdo con los principios aplicables a cada espacio.

A través de estas herramientas se construye un espacio de visibilidad, impacto y reconocimiento desde 
el cual se podrían fortalecer relaciones de trabajo, proyectos y programas de intervención. Su aplicación debe concebir los principios articuladores de cada espacio social; de ahí que no sería posible explicar sus resultados sin entender el habitus: las "estructuras estructuradas, principios generadores de prácticas distintas y distintivas" y las "estructuras estructurantes, principios de clasificación"(Bourdieu, 1998, p.15). A su vez, todo parece indicar que los estudios métricos resultan igualmente útiles para dar cuenta del habitus, dado que por un lado es una "estructura estructuradora" - es decir, una estructura que estructura el mundo social (Ritzer, 1993) y constituye una guía para la acción, la percepción y el procesamiento de la información -y, por otro, es una "estructura estructurada", es decir, una estructura estructurada por el mundo social (Ritzer, 1993).

Resulta indispensable diferenciar cuando se intenta evaluar la ciencia desde una posición crítica y académica como la que se puede generar en las universidades, sin ningún efecto más allá del ámbito discursivo, de cuando lo hacen los gestores científicos con fines e impacto económicos en el sueldo de los científicos. En el primer caso, las herramientas son refinadas y las conclusiones pueden estar llenas de matices. En el segundo, es simplemente una tosca herramienta de corte que separa los que se merecen algo en concreto de los que no.

Se ha tener suma precaución en la utilización y complementariedad con otras técnicas y procedimientos evaluativos. La proliferación de indicadores bibliométricos en años recientes ha generado cierto tipo de desorientación entre los tomadores de decisiones quienes a la larga no han sido capaces de determinar los pros y los contras del uso de varios de esos indicadores en la planificación del ejercicio evaluativo actual. El desempeño debe evaluarse con respecto a las metas específicas y los objetivos trazados. Por consiguiente, los objetivos deben declararse en condiciones mensurables que representen el resultado deseado de la actividad científica. El principal indicador de desempeño de una unidad de producción (sea este un individuo, grupo de investigación, departamento, institución, campo, disciplina, región o país) es la proporción entre el valor del rendimiento de la producción de salida y el valor de las entradas requeridas para producirlo (Abramo \& D’Angelo, 2014).

Investigaciones realizadas (Delgado \& Repiso, 2013; De-Filippo, 2013; Mañana \& Sierra, 2013) corroboran que los elementos anteriormente expuestos son aplicables a la investigación que se genera en las Ciencias Sociales de manera general y en la Comunicación en lo particular. No obstante, se necesitan muchas más investigaciones en aras de realmente comprender las complejidades involucradas en estos espacios (Moed, 2011).

Su evaluación se ha convertido en un área cada vez más atrayente. En las ciencias humanas y sociales existe un imponderabley es que los resultados encontrados por la utilización de determinados indicadores, si bien pueden fortalecer tendencias, deben ser analizados en un contexto para evitar riesgos de interpretaciones erróneas o injustas, porque no tiene mucho sentido emitir declaraciones sobre las características de las Ciencias Sociales y Humanidades como un todo (Mugnaini et al., 2006; Moed, 2011).

En esta área la investigación manifiesta una marcada orientación hacia lo local (Archambault et al., 2006; Žic Fuchs, 2014) y la cultura de comunicación científica suele variar de una disciplina a otra, aunque de manera general los resultados se trasmiten a través de monografías y compilaciones. Sin embargo, en los últimos tiempos se ha evidenciado un aumento del número de artículos publicados por parte de investigadores de estos espacios en revistas científicas, posiblemente por el hecho de que los resultados investigativos asociados a dichos investigadores, sus grupos o instituciones están siendo evaluados a través de la contabilización del número de artículos publicados en revistas trascendentes (Osca-Lluch \& Haba, 2005).

Una característica histórica que avala los debates suscitados durante largo tiempo en torno al uso de índices de citas como el Social Science Citation Index y el Art \& Humanities Citation Index para la evaluación de las Ciencias Sociales y Humanidades radica en que el ahora denominado Thompson Reuters, nunca creó un Journal Citation Report para estos. Este particular reforzó la idea de que las Humanidades eran "inevaluables", al menos desde esta perspectiva. Por su parte, el Scimago Journal Rank brinda indicadores de todas las revistas en todas 
las categorías, un avance en el desarrollo de esta parte del conocimiento, y ha sido aceptado por la comunidad científica que en los últimos tiempos se hayan evidenciado mayores niveles de cobertura con respecto a estas áreas de conocimiento (Braun et al., 2000; Moya-Anegón et al., 2007).

Esta problemática, de carácter común para muchos países europeos y latinoamericanos en los últimos años, se ha venido afrontando a través del desarrollo de modelos de evaluación de revistas científicas con el concurso de diferentes indicadores de calidad, en aras de lograr su homologación, reconocimiento y visibilidad a nivel internacional. Desafortunadamente, lo que ha condicionado estos instrumentos no ha sido la propia "forma" de la ciencia sino más bien la "forma" y especialmente el tamaño que van tomando los grandes grupos concentrados, con Elsevier a la cabeza. Ellos están marcando la agenda de evaluación académica, a pesar de que hay iniciativas tales como la Declaración de San Francisco sobre Evaluación de la Investigación (DORA, San Francisco Declaration on Reseach Assessment) que intentan romper con ello, haciendo un llamado a reconocer la necesidad de mejorar la forma en la que se evalúan los resultados de la investigación científica. En el caso de las revistas de Comunicación, la tendencia a su inclusión en bases internacionales ha sido un proceso que, en los últimos años, ha afectado a todo el ámbito de las Ciencias Sociales (De-Filippo, 2013).

Si la metría tiene que ser incluida en la evaluación, entonces dicha evaluación debe ser informada por la metría, y no guiada por esta. La creación de bases de datos nacionales de revistas, monografías y otros productos de la investigación es el necesario primer paso para lograr una evaluación de la investigación en Humanidades saludable y enfocada en la calidad (Žic Fuchs, 2014); particular también extensivo a las Ciencias Sociales, pues estos espacios muchas veces son víctimas de la evaluación "fácil y barata" a fuerza de citas e impacto. Los gestores suelen funcionar muchas veces de forma mecánica, cayendo fácilmente ante la inmediatez que da la metría. Los intentos por evaluar la investigación sobre la base no solo de Scopus sino también del Web of Science manifiestan determinadas carencias, particularmente en el sentido de que estas fuentes no reflejan en muchos casos la verdadera calidad e innovación de las investigaciones en Ciencias Sociales y
Humanidades. Los niveles de cobertura aún son incipientes, particularidad que las coloca en total desventaja en comparación con otros dominios mayormente representados (Žic Fuchs, 2014).

Una breve mirada hacia datos concretos de España y Cuba, según el Scimago Journal \& Country Rank, corrobora el comportamiento anterior. Si bien el comportamiento de la distribución de revistas por grandes áreas es claramente muy diferente en ambos países (España 467 revistas, Cuba 24), estos coinciden en el protagonismo de la Medicina (España 152, Cuba 16). Llama la atención la cantidad de revistas de Ciencias Sociales y Humanidades españolas (142 y 125 respectivamente), mientras que Cuba cuenta solamente con 1 en la misma categoría. No obstante, se marca una tendencia al crecimiento que, en el caso de España, no es intuitiva a priori sino que evidentemente pudiera responder, entre otras cuestiones, a las políticas y estrategias que se han venido implementando en el país para validar la ciencia. Siguiendo la propuesta de Moya-Anegón y Herrero-Solana (2013) es posible afirmar que, a partir de la producción científica con visibilidad internacional, Cuba se adscribe a un modelo que quizás lo distingue del que poseen otros países de América Latina y el Caribe. Se encuentra bajo el influjo de un modelo claramente socialista pero con un dominio temático dominado por el paradigma de la Biomedicina y en detrimento de la investigación social.

Si bien se destacan otras alternativas, la Agencia Nacional de Evaluación y Prospectiva de España estableció en el 2007 las guías básicas en las que sustentar la evaluación de las diferentes actividades investigadoras en este ámbito, partiendo del presupuesto de que la inversión española en investigación científica debe tener una correspondencia en la productividad de los investigadores en el plano internacional. Un ejemplo claro de este particular es la publicación reciente de los criterios para evaluar los "sexenios" o complementos de investigación en España de acuerdo a la Resolución de 26 de noviembre de 2014, de la Comisión Nacional Evaluadora de la Actividad Investigadora (CNEAI). Las condiciones en general se han endurecido para la mayor parte de las ciencias, salvo las Ciencias Sociales (comisión 7). En esta comisión se aplica un criterio relativamente laxo para todas las Ciencias Sociales (incluida la 
Comunicación), salvo para dos disciplinas: Psicología y Biblioteconomía y Documentación (ByD) (España, 2014). Es la primera vez que nuestra disciplina aparece claramente reflejada y lo es para poner el listón evaluativo más alto. Es posible asegurar que no serían muchos los que identificarían a la ByD como una de las disciplinas sociales con mayor rigor científico; puede que muchos acierten la primera, pero nadie acertaría la segunda. Esta es una clara interpretación de la ciencia con un enfoque "administrativo".

Uno de los rasgos distintivos de la actividad científica cubana en el campo de las Ciencias Sociales y Humanísticas, protagonizada por las instituciones de Educación Superior adscritas al Ministerio de Educación Superior, radica en la correspondencia de la actividad de las investigaciones socio-humanísticas con las demandas y necesidades sociales (Morejón Seijas, 2007).

Dichas demandas y necesidades sociales han sido formalmente reconocidas a través de la política científica como problemas priorizados de investigación de diferentes niveles jerárquicos que precisan ser abordados y/o resueltos por la comunidad científica. Se habla entonces de los Programas de Ciencia e Innovación Tecnológica de carácter socio-humanístico, tanto a nivel nacional, ramal como territorial, y también de las 14 áreas de resultados clave o prioridades contenidas en la Política Científica de las Ciencias Sociales y Humanísticas en el país. Sin embargo, la Comunicación Social no queda correctamente contemplada en este marco regulatorio.

Sobre la base de los anteriores supuestos y teniendo en cuenta que se aboga por un tránsito hacia un modo 2 de producción de conocimiento, según el cual las formas de evaluación de la calidad han de ser más integrales y capaces de sobrepasar los criterios de autoridad tradicionales basados en la evaluación de pares y las publicaciones (Gibbons et al., 1994), se hace un llamado a la implementación y desarrollo sustancial de estos aspectos de manera general en el campo de la investigación en nuestro país y, específicamente, en el de la Comunicación.

\section{Condicionantes para la evaluación de la investigación cubana en Comunicación Social}

Como un fenómeno complejo, plurideterminado, estudiado desde las más diversas disciplinas, que involucra todas las esferas de la sociedad y se encuentra estrechamente relacionado con el poder político, económico, académico y personal (Benítez Arenas, 2012), el pensamiento comunicacional iberoamericano puede ser valorado como periférico, subalterno o negado por las propias lógicas de evaluación y planeación nacionales, cuyos parámetros y criterios vienen marcados por dos erráticas lógicas o principios: la uniformidad entre campos, disciplinas y países y la racionalidad instrumental orientada a resultados y productos según culturas de investigación y visiones positivas foráneas que inciden o afectan negativamente el impulso y visibilidad de los circuitos, espacios y producción autóctona (Sierra Caballero, 2012).

Según (Sierra Caballero, 2012, p.3), una de las principales contradicciones internas de la propia política científica de nuestros países es que

$$
\begin{aligned}
& \text { [...] ha renunciado a la coherencia y } \\
& \text { sostenibilidad necesarias para el desarrollo social, } \\
& \text { aceptando sistemas de copyright, modelos de } \\
& \text { evaluación y políticas de productividad inviables } \\
& \text { por escasez de recursos, sentido común y } \\
& \text { pertinencia desde el punto de vista del desarrollo } \\
& \text { endógeno. Se observa con frecuencia el dominio } \\
& \text { de una lógica de organización de la actividad } \\
& \text { científica dispersa y carente de un espacio propio } \\
& \text { definido y claramente acotado, lo que viene } \\
& \text { repercutiendo de forma adversa en la evaluación } \\
& \text { por parte de las más altas autoridades políticas } \\
& \text { de la gestión pública de la política científica. }
\end{aligned}
$$

Todo indica que, para proponerse la reestructuración del trabajo científico en el campo de la comunicación, resulta clave comprender sus marcas institucionales de desarticulación múltiple. Una de las claves para remontar colectivamente la situación de fragmentación y estancamiento podría encontrarse en el reconocimiento de la creciente irrelevancia social que detectan algunos investigadores líderes en la investigación de la comunicación (Fuentes Navarro 2010). El campo enfrenta tres desafíos principales: la pérdida de identidad, la erosión epistemológica y la falta de relevancia social de la investigación. La investigación de la comunicación tiene el potencial y el deber de enfocarse en agendas de investigación que puedan ayudar a las sociedades a tomar decisiones sobre cualquier asunto, a partir de una sólida base de evidencias y con la menor influencia posible de otras personas o instituciones (Donsbach, 2006). 
En este sentido, para la consolidación y/o estructuración de un campo es determinante abordar sus prácticas, nivel de reflexividad y los nexos que se establecen entre los agentes que lo estructuran, lo construyen y deconstruyen. En el campo de la Comunicación a nivel iberoamericano se vienen desarrollando un conjunto de acciones que parecen reconocer la notoriedad de la evaluación de la investigación. Este comportamiento se corrobora en el Manifiesto Programa de la Confederación Iberoamericana de Asociaciones Científicas en Comunicación (CIAC), más conocido como Manifiesto Programa Funchal. Concretamente, se propuso la articulación de nuevas bases de progreso y el diseño de una estrategia de convergencia que garanticen una representación y articulación política interna y externa en materia de política científica; la definición de una política de cooperación basada en formas flexibles, autónomas y coordinadas en red; la producción de información y conocimiento regional sobre nuestras realidades científicas y académicas y la constitución de instrumentos propios de organización que representen y validen el campo comunicacional iberoamericano (Confederación Iberoamericana de Asociaciones Científicas en Comunicación, 2009). En el plan de trabajo concebido hasta el año 2014 se contempló, en el acápite concerniente a la investigación, un conjunto de tareas que tributaron al desarrollo y cohesión del campo en cuestión. Se propuso de esta manera elaborar un listado de revistas y publicaciones científicas; el diseño de un mapa de grupos e investigadores del campo comunicacional iberoamericano; la identificación de indicadores y criterios de validación y calidad comunes de la producción científica regional; documentación científica en bases de datos integradas de proyectos de I+D (investigación + desarrollo), centros de educación superior y posgrados de especialización regionales así como la organización de encuentros sobre política científica regional y la sistematización de la producción científica en temáticas, generales y especializadas de comunicación.

En esta misma línea, en el documento de trabajo del I Foro de política científica y tecnológica en Comunicación celebrado en 2012, quedó evidenciada la pertinente y prioritaria necesidad de un Plan Estratégico 2012-2020 del Espacio Iberoamericano de
Investigación en Comunicación que contemple: la redacción de un Libro Blanco Iberoamericano de Investigación en Comunicación que evalúe, diagnostique y apunte, prospectivamente, líneas de futuro de la producción científica regional; un Plan de Formación de Cultura de Investigación que priorice el estudio y conocimiento del pensamiento latinoamericano y promueva programas de altos estudios sobre las nuevas fronteras del conocimiento comunicacional; una política de publicaciones y catálogo de recursos y redes de difusión del conocimiento (Sierra Caballero, 2012).

Por su parte, la investigación en Comunicación en Cuba atiende a regularidades propias de las tendencias internacionales en este campo, aunque presenta particularidades que sobresalen en el contexto regional. A nivel nacional no se cuenta con un plan de desarrollo en torno a la investigación en comunicación salvo aquel que las propias escuelas de comunicación y algunas instancias nacionales como las Comisiones Nacionales de Carrera han logrado impulsar. Sin embargo, la práctica profesional de la Comunicación Social ha venido extendiéndose estructuralmente en todo el país, proceso en el cual la formación de profesionales y los resultados de investigaciones de tesis han tenido un papel significativo.

El país se encuentra inmerso en una etapa de transformaciones y reformas proyectadas a partir de la consulta popular y el consenso democrático en torno a los Lineamientos de la Política Social y Económica aprobados en el VI Congreso del Partido Comunista de Cuba (PCC) . Este proceso de reordenamiento ha tenido una prioridad en el área económica, lo cual comienza a tener una incidencia en el resto de los componentes del sistema social (Partido Comunista de Cuba, 2011).

Aunque en el país existe un marco normativo para la actividad científica dirigido por el Ministerio de Ciencia, Tecnología y Medio Ambiente, en el área de la comunicación la normatividad es dispersa y limitada. De notable incidencia profesional es la Resolución 60 del año 2011 de la Contraloría de la República, en la cual se identifican la información y la comunicación entre los componentes transversales del control interno así como el anuncio de la creación de un Grupo no Permanente de la Comisión de Implementación de los Lineamientos para trabajar en una propuesta de Política de Comunicación del Gobierno y el Estado Cubano. 
Otros esfuerzos en sentido normativo para el sector empresarial son la aprobación del Decreto-Ley nº 252 (Cuba, 2007a) Sobre la continuidad y el fortalecimiento del sistema de dirección y gestión empresarial cubano y el Decreto no 281 (Cuba, 2007b) Reglamento para la implantación y consolidación del Sistema de Dirección y Gestión Empresarial Estatal, reguladores del denominado Sistema de Perfeccionamiento Empresarial, que comprende un subsistema dedicado íntegramente a la comunicación.

La investigación de la comunicación no cuenta con políticas claras para su proyección y articulación en un contexto social de producción y socialización amplia de conocimientos. Sin embargo, la investigación que se realiza se concibe tanto para la producción de conocimientos teóricos y empíricos como para la búsqueda de referentes e información necesarios para la producción comunicativa (Saladrigas Medina \& Olivera Pérez, 2012) lo cual ha venido acumulando un impacto social, institucional y cultural nada despreciable pero carente de medición.

Cuba manifiesta un comportamiento atípico que lo diferencia del resto de los países de la región y del mundo toda vez que, por un lado, el campo investigativo cubano se encuentra permeado por normas establecidas por los organismos científicos que controlan y regulan el proceso de investigación y formación; y, por otro, se distingue de la realidad regional e internacional al concebir la actividad investigativa bajo un régimen del cual no se ha beneficiado. Se considera que ha sido un objeto ignorado y tan inmerso dentro de otras prácticas que la sociedad no siente la necesidad de investigar "la Comunicación" aun cuando sí se investiga ${ }^{4}$. Este comportamiento trae consigo la falta de reconocimiento en torno a la existencia de un campo propio, mientras que quienes lo reconocen, lo analizan como un campo centrado en las formas profesionales de hacer la Comunicación pero no como espacio capaz de legitimarse científicamente a partir de pensarse y autorreflexionarse. En este sentido se han logrado identificar algunas de las problemáticas fundamentales (Saladrigas Medina \& Olivera Pérez, 2010): no resulta un área disciplinar a considerar dentro de las ciencias sociales, por lo tanto no se le concibe con necesidades de índole científico; pobre intercambio de información y conocimiento producido entre los tres sub-campos que lo constituyen y dentro de cada uno ellos en particular; falta de una política de organización y socialización de la información y el conocimiento producido; cooperación débil y parcelada aun cuando existen mecanismos de intercambio y trabajo entre las diferentes estructuras; la construcción de conocimientos sigue patrones establecidos tradicionalmente por las atribuciones institucionales, con lo cual se precisa la búsqueda de nuevas formas de concreción; el conocimiento creado no está debidamente atesorado, ni organizado.

Aun cuando estas necesidades son identificadas a su vez por varios agentes del campo, la propia desarticulación, las habituales rutinas laborales, particulares conflictos y el déficit infraestructural de nuestras instituciones docentes e investigativas se cuentan entre los obstáculos con los cuales tendría que lidiar este proyecto. Favorablemente se acumulan relaciones de trabajo, vínculos interinstitucionales, un mínimo de condiciones técnicas y la voluntad de articulación de un grupo de agentes, entre los más interesados: el Centro de Investigaciones Sociales del Instituto Cubano de Radio y Televisión, la Asociación Cubana de Comunicadores Sociales, la Facultad de Comunicación de la Universidad de La Habana, y los Departamentos de Periodismo y Comunicación Social de las Universidades de Pinar del Río y Central de Las Villas.

Asimismo, se registran varios intentos y espacios de coordinación como el Encuentro Internacional de Profesionales de la Información y la Comunicación, la Revista Alcance de Información y Comunicación y la débil estructura del Círculo de Investigadores de la Comunicación de la Asociación Cubana de Comunicadores Sociales.

Estos elementos, junto a las reflexiones teórico-epistemológicas necesarias, hacen evidente la urgencia e importancia de valorar la investigación científica que se desarrolla en el campo con el objetivo de obtener una

\footnotetext{
${ }^{4}$ Según los registros en bases de datos resultado de tesis de diploma y maestría realizados en los últimos años como parte de la línea de investigación "Campo Académico y Profesional de la Comunicación en Cuba". Solo entre los años 2006 y 2011 se registran en las principales instituciones de formación universitaria del país cerca de 1000 investigaciones de tesis en el área de la comunicación (Hernández Barrera, 2015).
} 
visión holística de este espacio como dominio de conocimiento a partir de la identificación, descripción e interpretación de las agendas investigativas y la estructura intelectual.

La evaluación de la investigación debe convertirse en acápite clave para el meta-análisis de la ciencia. La consecuente sistematización de la aún dispersa producción científica nacional sobre Comunicación Social así como el subsiguiente diseño y aplicación de una amplia batería de indicadores basados previamente en la producción, la colaboración y, en la medida de lo posible, el impacto y visibilidad a tono con las características y referentes antes subrayados contribuiría a caracterizar, desde este punto de vista, el dominio de manera general, sus patrones de relaciones colaborativas y la estructura intelectual del campo y, con ello, explicar los esquemas de comportamiento a partir de los elementos contextuales provenientes de su estructura histórica y epistemológica.

La consecuente imbricación de estos aspectos permitirá entonces proponer las pautas, estrategias y agendas que bien pudiesen ser parte formativa o tomarse como referente para la generación de políticas científicas en pos de incentivar la investigación, su correcta gestión y el desarrollo de este campo tanto a nivel teórico como práctico en nuestro país.

Queda claro que el subcampo investigativo constituye uno de los principales puntos de mira toda vez que está conformado esencialmente por dos tipos de centros: las instituciones educativas y los centros y/o departamentos de investigación.

Las instituciones educativas que realizan proyectos de investigación como ejercicio de práctica o graduación de sus estudiantes, en sus dos modalidades de enseñanza (pregrado y posgrado), son las que llevan el mayor peso en dicha producción. En Cuba existen algunos centros de investigación que, sin tener como objeto de indagación a la comunicación, la incorporan a sus estudios, al menos desde alguna de sus aristas. Ello obedece, como se apuntó anteriormente, a que la Comunicación es un fenómeno que atraviesa múltiples espacios sociales y ha sido históricamente abordado desde diferentes áreas disciplinares (Benítez Arenas, 2012).
Vale destacar que la exigencia de realizar diagnósticos como parte del subsistema de comunicación del Perfeccionamiento Empresarial y las más recientes solicitudes para la asesoría en la elaboración de las políticas gubernamentales y sus respectivas estrategias de comunicación han demandado la realización de investigaciones de alto impacto social y económico como parte de las Prácticas Laborales, los trabajos de diploma o de maestría de la Facultad de Comunicación de la Universidad de La Habana y otros departamentos docentes del país.

Se trata de una investigación de tipo aplicada, para la resolución de problemas concretos, que ha permitido ganar un espacio importante de reconocimiento para la profesión. Reporta múltiples beneficios: los estudiantes aprenden en la práctica y se entrenan; los profesores tienen material empírico para luego realizar reflexiones teóricas y aportes al Programa del plan de estudio; las empresas dan respuesta a sus necesidades concretas con personal calificado; el país se desarrolla. Es perceptible una articulación entre los tres subcampos (académico, científico y profesional) a través de ciclos de producción-innovación-desarrollo que deben ser conducidos con mayor coherencia.

La producción científica en el espacio académico está fuertemente asociada al desarrollo, vaivenes y retrocesos en la formación profesional de periodistas y comunicadores sociales (Saladrigas Medina \& Olivera Pérez, 2009) y marcada por las características propias de la investigación de pregrado: la inexperiencia e inmadurez teórica de los alumnos-investigadores, la carencia de recursos materiales para apoyar el trabajo, el carácter unipersonal de los esfuerzos, el enfoque unidisciplinar y el bajo grado de sistematización y socialización de los resultados (Benítez Arenas, 2012). Aun así, algunos trabajos han cumplido requisitos muy superiores a los exigidos para ese nivel, pasando de estudios puramente descriptivos y reproductivos a investigaciones analíticas de fenómenos cada vez más globales y diversos de la comunicación (Saladrigas Medina \& Olivera Pérez, 2009).

Debe destacarse que uno de los programas de formación académica de mayor tradición en el país es la Maestría en Ciencias de la Comunicación, la cual cuenta con catorce ediciones abiertas y se viene desarrollando 
desde el año 1994. Además, el sistema Nacional de Formación de Doctorado ha propiciado la graduación de más de 40 doctores en Ciencias de la Comunicación, varios de ellos provenientes del sector profesional, lo cual constituye una tipicidad de Cuba que no resulta común en el resto de la región latinoamericana y caribeña.

Estudios conducidos en la línea "Campo Académico y Profesional de la Comunicación en Cuba" de la Facultad de Comunicación de la Universidad de La Habana constituyen uno de los esfuerzos significativos de investigación dentro de dicha institución que ha contribuido en la difícil tarea de caracterizar la investigación producida desde 1970 y hasta la primera década de 2000 (Saladrigas Medina, 2005; Salas Hernández \& Olivera Pérez, 2006; Muñoz Nieves \& Olalde Azpiri, 2010; Benítez Arenas, 2012; Hernández Barrera, 2015).

Acumula más de 30 tesis de pregrado y posgrado, una veintena de artículos y ponencias y varias propuestas innovadoras para la modificación de planes de estudio u otras ofertas científico-docentes.

Dentro de la mencionada línea se han desarrollado iniciativas de reconstrucción sociológica y crítica epistemológica en torno al campo de la Comunicación, que se han articulado a partir de la reflexión latinoamericana al respecto así como de los esfuerzos aislados que, en este sentido, se habían realizado en el país en décadas anteriores. Para ello se ha ido conformando una base de datos con la información de la memoria escrita de las investigaciones del campo, fuente que, con el debido tratamiento, normalización y plataforma tecnológica, se constituye como referente para aplicar los principios evaluativos de la investigación científica en el campo desde una perspectiva cienciométrica.

Ante una investigación realizada fundamentalmente a partir de esfuerzos individuales y de líneas poco claras e insuficientemente desarrolladas - en muchos casos no asociada a las formas institucionales de gestión del trabajo científico que desarrollan otras áreas de las ciencias sociales, no inscrita casi nunca en programas nacionales, ramales o territoriales ni reflejada en proyectos que las hagan impactantes y sustentables; con una agenda muy puntual y generalmente espontánea en centros de investigación social y facultades afines en las universidades; con ausencia de un centro de investigación que integre los diferentes espacios donde se da el fenómeno; falta de estrategias pertinentes para la inserción de los comunicadores sociales en espacios investigativos; incremento en la publicación de textos de autores nacionales a la vez que una diversificación de las temáticas tratadas en torno a la comunicación en detrimento de la producción, publicación y socialización de artículos científicos debido a la deficiencia de espacios nacionales; junto a la ausencia de una política definida en torno al atesoramiento, organización, conservación de la información científica producida en el campo y que atenta contra el asentamiento del patrimonio de conocimientos en materia de Comunicación Social en el país (Benítez Arenas, 2012), se impone asumir el reto de intencionar más las colaboraciones y sistematizar los resultados disponibles para hacer una parada autorreflexiva en aras de corroborar la caótica realidad, evaluarlos más concretamente y, a partir de ahí, generar las soluciones necesarias en términos de gestión correcta de la investigación del campo en cuestión.

Los elementos anteriores apuntan entonces a la necesidad de una política científica coherente e integrada que conciba por etapas la superación y el rigor investigativo, articule el posgrado y el pregrado, redefina líneas de investigación cuya gestión se sustente en principios como la flexibilidad, la cooperación y el respeto en el trabajo y la coordinación, oriente la investigación hacia la gestión de proyectos, potencie estrategias de publicación, integre las demandas de la docencia y la producción científica y asuma el reto académico de la enseñanza transversal de la investigación, para lograr estar en total correspondencia con los modelos y teorías actuales que regulan la producción social de conocimientos.

Aun cuando las características puntuales de la investigación en el campo se agrupan en los criterios antes mencionados, un modelo que articule la evaluación de la investigación en este ámbito en nuestro país aún está por determinarse; hasta este punto solo ha sido posible sistematizar la investigación de este dominio a partir de su muy disgregada producción científica.

De manera que se precisa realizar un número mayor y más frecuente de investigaciones con el concurso de diferentes fuentes de datos (nacionales e internacionales 
en la medida de lo posible), atendiendo a diferentes realidades y niveles de agregación.

Urge ser prudentes en la utilización de las diferentes herramientas disponibles para el desarrollo de la evaluación, que en este espacio contribuirá a elevar el nivel de reconocimiento del desempeño de los científicos sociales - y, con ellos, los comunicadores -, examinar la dualidad existente entre el conjunto de actividades sociales y económicas y palear un tanto la incoherencia disciplinaria y, al mismo tiempo, el nivel de marginalidad que se le atribuye dentro de las Ciencias Sociales y de estas a su vez dentro de la ciencia de manera general.

Este esfuerzo debe encaminarse paralelamente con la identificación de invariantes y de los principios que sustentan la práctica comunicativa y la práctica científica en la sociedad cubana, la cual ha tenido en las últimas seis décadas una clara marca de inclusión social, acceso y socialización del conocimiento y la cultura.

Como diría Sierra (2012, p.5), "hoy es el momento de confrontar el reto de la globalización en la era del Capitalismo Cognitivo y empoderar la praxis y cultura de investigación regional [...] O nos organizamos como campo o nos organizan las líneas, agendas y formas de investigación". Al reflexionar sobre este particular es posible afirmar que, en el contexto nacional y con respecto al campo en cuestión, la aplicación de los elementos expuestos es más que una necesidad imperante: es una realidad preponderante toda vez que los elementos necesarios para su articulación están dispuestos, sin embargo, se impone lograr articularlos correctamente. Ese es el reto.

\section{Conclusión}

La cultura de la evaluación ha invadido disímiles entornos en los últimos 20 años. En el ámbito universitario ha marcado pauta de manera mayoritaria, pues la evaluación de la ciencia contribuye a emitir criterios relativos a la efectividad de las políticas científicas nacionales en pos de impulsar el avance del potencial científico-investigativo así como a la identificación de debilidades y fortalezas de los actores implicados en dicha actividad. Se erige como instrumento para el establecimiento de prioridades el nivel de cumplimiento de los objetivos trazados originalmente, el valor y relevancia de sus resultados, así como los factores que determinan la obtención o no del éxito. Se realiza en múltiples entornos, con la anuencia de varios agentes y recurriendo a disímiles criterios y herramientas de evaluación.

Urgen investigaciones que, desde la perspectiva de las Ciencias de la Información, sus técnicas, herramientas y experiencia, tributen a visualizar la importancia de la gestión de la ciencia y, a partir de ahí, el cómo crear una cultura de la necesidad de gestionarla para el campo de la Comunicación en el contexto cubano, en pos de obtener herramientas sólidas que promuevan su desarrollo.

Para contribuir a la estructuración del campo de la Comunicación Social en Cuba así como a una correcta gestión de la investigación que se genera se requiere, junto a las reflexiones teórico-epistemológicas necesarias, abordar sus prácticas, su nivel de reflexividad y los nexos que se establecen entre sus protagonistas. Si bien aún modestos, en nuestro país se están dando pasos de avance en este sentido.

Los elementos inherentes a la evaluación de la investigación aplicados a este espacio junto al concurso de herramientas propias de la metría de la información marcarían pauta en la identificación, descripción e interpretación de las agendas investigativas y su estructura intelectual, permitiendo obtener una mirada holística de este dominio de conocimiento. La consecuente imbricación de todos estos elementos permitirá proponer directrices, estrategias, lineamientos, modelos y programas para encauzar la necesaria Política Científica Nacional de Comunicación.

La evaluación de la investigación en este campo no resulta tarea sencilla y se ve afectada, a priori, por condicionantes y retos que deberán ser superados para obtener resultados óptimos. Se habla, entre otras cuestiones, de:

- Ausencia de una idónea cultura investigativa a nivel de campo de la Comunicación Social en el país: que la tenga un pequeño grupo de sujetos no significa que la tenga todo el campo; un marcado carácter profesionalizante que no reconoce el valor de la investigación teórico-histórica del campo en general (profesional-académico- 
-investigativo) aun cuando se realiza; no se reconoce la existencia de un campo propio y, cuando se reconoce, se visualiza como un campo de "haceres" y no de "pensares".

- Incipiente existencia de publicaciones nacionales del campo en cuestión y, por consiguiente, nula o casi nula visibilidad internacional de la producción científica nacional a partir de fuentes propias, si bien se tiende a publicar en revistas extranjeras.

- Inexistencia de un modelo de gestión de la investigación y de un sistema de información científica, concebido con condiciones tecnológicas idóneas que permitanagrupar, sistematizary regular metodológicamente toda la producción científica nacional en aras de que sirva como referente para la gestión y evaluación de la actividad científico-investigativa del campo así como la consecuente socialización a diferentes niveles.

- Se impone la concepción de una sólida batería de indicadores en total consonancia con la realidad, las características del dominio en cuestión y con las proyecciones de políticas públicas en las áreas de la información, la comunicación, la educación y la cultura que ha proyectado o prevé proyectar el gobierno nacional.

\section{Colaboraciones}

Todos los autores contribuyeron en la concepción, diseño del estudio, análisis de datos y redacción final.

\section{Referencias}

Abramo, G.; D'Angelo, C.A. Research evaluation: Improvisation or science? In: Bibliometrics: Use and abuse in the review of research performance, v.87, 2014. (Wenner-Gren International Series). Available from: <http://www.portland press.com/pp/books/online/wg87/087/0055/087 0055.pdf>. Cited: Sept. 15, 2014.

Archambault, E. et al. Benchmarking scientific output in the social sciences and humanities: The limits of existing databases. Scientometrics, v.68, n.3, p.329-342, 2006. Available from: <http://www.akademiai.com/index/34w733263n36 h661.pdf>. Cited: Sept. 15, 2014.

Arencibia, R. Sistematicidad en la evaluación de la actividad científica desde una perspectiva cienciométrica. Acimed, v.23, n.3, 2012. Disponible en: <http://acimed.sld.cu/index.php/ acimed/article/view/390/233 > . Acceso en: 15 sept. 2014.

Arencibia, R.; Moya Anegón, F. La evaluación de la investigación científica: una aproximación teórica desde la Cienciometría. Acimed, v.17, n.4, 2008. Disponible en: <http:// goo.gl/nB2rmw>. Acceso en: 15 sept. 2014.

Benítez Arenas, L. Una mirada al campo de la comunicación social en los albores del siglo XXI. 2012. Tesis (Maestría en Ciencias de la Comunicación) - Universidad de La Habana, La Habana, 2012.

Bourdieu, P. Capital cultural, escuela yespacio social. México: S. XXI, 1998

Braun, T.; Glanzel, W.; Schubert, A. How balanced is the Science Citation Index's Journal coverage? A preliminary overview of macrolevel satistical data. In: Cronin, B.A. (Ed.). The Web of Knowledge: A festschrift in honor of Eugene Gardfield. Ottawa: American Society of Information Science, 2000. p.251-277.

Confederación Iberoamericana de Asociaciones Científicas en Comunicación. Manifiesto Programa IBERCOM. 2009. Disponible en: <http://www.ulepicc.es $>$. Acceso en: 22 sept. 2014.
Cuba. Consejo de Ministros. Decreto-Ley no 252, de 7 de agosto de 2007. Sobre la continuidad y el fortalecimiento del sistema de dirección y gestión empresarial cubano. Gaceta Oficial de la República de Cuba, Ministerio de Justicia, Habana, 7 agosto 2007a. p.160. Disponible en: <http://www.economiasnci.gob.mx/politicacomercial/archivos/Decreto\%20281-\% 202013.pdf>. Acceso en: 22 sept. 2014.

Cuba. Consejo de Ministros. Decreto no 281, de 16 de agosto de 2007. Reglamento para la implantación y consolidación del Sistema de Dirección y Gestión Empresarial Estatal. Gaceta Oficial de la República de Cuba, Ministerio de Justicia, Habana, 16 agosto 2007b. p.160. Disponibleen: <http://www.economiasnci.gob.mx/politicacomercial/archivos/Decreto\%20281-\% 202013.pdf>. Acceso en: 22 sept. 2014

De-Filippo, D. La producción científica española en comunicación en WoS: las revistas indexadas en SSCI (200712). Comunicar: Revista Científica de Educomunicación, v.XXI, n.41, p.25-34, 2013. Disponible en: <http://www.revista comunicar.com/index.php?contenido=revista\&numero $=41>$. Acceso en: 15 sept. 2014.

Delgado, E.; Repiso, R. El impacto de las revistas de comunicación: comparando Google Metrics, Web of Science y Scopus. Comunicar: Revista Científica de Educomunicación, v.XXI, n.41 p.45-52, 2013. Disponible en: <http://www.revista comunicar.com/index.php?contenido=revista\&numero= 41>. Acceso en: 15 sept. 2014.

Donsbach, W. The identity of communication research. Journal of Communication, v.56, n.3, p.437-448, 2006.

España. Comisión Nacional Evaluadora de la Actividad Investigadora. Resolución de 26 de noviembre de 2014. Criterios específicos aprobados para cada uno de los campos de evaluación. Boletín Oficial del Estado, España, 2014. Disponible en: <https://www.boe.es/boe/dias/2014/12/01/ pdfs/BOE-A-2014-12482.pdf>. Acceso en: 22 dic. 2014. 
Fuentes Navarro, R. Investigación de la comunicación: referentes y condiciones de un diálogo transversal de saberes. Signo y Pensamiento, v.29, n.57, p.38-49, 2010.

Gibbons, M. et al. The new production of knowledge: The dynamics of science and research in contemporary societes. Thousand Oaks: Sage Publications, 1994.

Hernández Barrera, Y. Trazando el mapa: análisis del campo investigativo de la comunicación en la Facultad de Comunicación de la Universidad de la Habana en el periodo comprendido entre los años 2005 y 2014. 2015. Tesis (Diploma en Periodismo) - Universidad de La Habana, 2015.

Mañana, J.; Sierra, B. La multidisciplinariedad de las revistas de comunicación españolas y extranjeras. Comunicar: Revista Científica de Educomunicación, v.21, n.41, p.71-81, 2013. Disponible en: <http://www.revistacomunicar.com/ index.php?contenido=revista\&numero $=41>$. Acceso en: 15 sept. 2014

Moed, H.F. Research assessment in social sciences and humanities. In: Ecoom Colloquium: Assesing Research Performance in the Social Sciences and Humanities, 2011, Antwerp. Electronic Proceedings... Antwerp: University of Antwerp, 2011. Available from: <http://goo.gl/ftRbR8>. Cited: Sept. 15, 2014

Morejón Seijas, B. Investigaciones sociales y humanísticas en las instituciones del MES: diagnóstico para el perfeccionamiento de su gestión. Novedades en Población: Revista Especializada en Temas de Población, v.3, n.5, p.189-279, 2007.

Moya-Anegón, F.; Herrero-Solana, V. Worldwide topology of the scientifc subject profile. PLoS One, n.9, 2013. Available from:<doi:http://dx.doi.org/10.1371/journal.pone.0083222>. Cited: Sept. 15, 2014.

Moya-Anegón, F. et al. Coverage analysis of Scopus: A journal metrci approach. Scientometrics, v.73, n.1, p.53-78, 2007. Available from: <doi:http://dx.doi.org/10.1007/s11192-007-16 81-4>. Cited: Sept. 15, 2014

Mugnaini, R., De Carvalho, T.; Campanatti-Ostiz, H. Indicadores de produção científica: uma discussão conceitual. In. Aguiar Poblacion, D.; Porto Witter, G.; Modesto da Silva, J.F. (Org.). Comunicação \& produção cientifica: contexto, indicadores e avaliação. São Paulo: Angellara Editora e Livraria Ltda, 2006. p.315-340.

Muñoz Nieves, C.; Olalde Azpiri, M. Investigación en el campo académico de la comunicación: miradas inconclusas sobre un ayer inmediato: una aproximación desde Ciudad de La Habana entre los años 2005 y 2009. 2010. Tesis (Diploma en Periodismo) - Universidad de La Habana, 2010.

Núñez, J. Conocimiento académico y sociedad ensayos sobre política universitaria de investigación y posgrado. La Habana: Editorial UH, 2010.
Osca-Lluch, J.; Haba, J. Dissemination of Spanish social sciences and humanities journals. Journal of Information Science, v.31, n.3, p.230-237, 2005.

Partido Comunista de Cuba. Lineamientos de la política económica y social del Partido y la Revolución. In: Congreso del Partido Comunista de Cuba, 6., 2011, La Habana. Anales Electrónicos... La Habana: PCC, 2011. Disponible en: <http:// congresopcc.cip.cu/wp-content/uploads/2011/01/ documentos/proyecto-lineamientos-pcc.pdf>. Acceso en: 22 sept. 2014.

Ritzer, G. Teoría sociológica contemporánea. Madrid: Interamericana de España, 1993.

Saladrigas Medina, H. Coordenadas cubanas para un fenómeno complejo: fundamentos para un enfoque teóricometodológico de la investigación de la comunicación organizacional. 2005. Tesis (Doctoral en Ciencias de la Comunicación) - Universidad de La Habana, La Habana, 2005.

Saladrigas Medina, H.; Olivera Pérez, D. La investigación en la formación profesional del comunicador social cubano. In: Encuentro Latinoamericano de Facultades de Comunicación Social, 13., 2009, La Habana. Anales... La Habana: Universidad de La Habana, 2009. p.844-885.

Saladrigas Medina, H.; Olivera Pérez, D. Emergencia de una red de conocimientos en el campo académico de la comunicación social en Cuba. In: Congreso Internacional de Información-INFO, 2010, La Habana. Anales... Habana: EDICT, 2010. p.12-18.

Saladrigas Medina, H.; Olivera Pérez, D. La investigación en comunicación: su lugar en Cuba. Revista Redes.Com, n.6, p.343-363, 2012. Disponible en: <http://revista-redes. hospedagemdesites.ws/index.php/revista-redes/article/ view/210>. Acceso en: 22 sept. 2014.

Salas Hernández, J.; Oliveira Pérez, D. Pasión y prejuicio: acercamiento histórico a la investigación en comunicaion social en Cuba (1970-1989). 2006. Tesis (Diploma em Comunicación) - Universidad de La Habana, La Habana, 2006.

Sierra Caballero, F. Documento de trabajo 2012. In: Foro de Política Científica y Tecnológica en Comunicación, 1., 2012, Quito. Anales electrónicos... Quito: Confibercom, 2012. Disponible en: <www.compoliticas.org>. Acceso en: 15 sept. 2014.

Van Raan, A.F. Advances in bibliometric analysis: Research performance assessment and science mapping. In: Bibliometrics: Use and abuse in the review of research performance. London: Performance Press, 2014. (Wenner-Gren International Series, v.87). Available from: <http:// www.portlandpress.com/pp/books/online/wg87/087/0017/ 0870017.pdf>. Cited: Sept. 22, 2014.

Žic Fuchs, M. Bibliometrics: Use and abuse in the humanities. In: Bibliometrics: Use and abuse in the review of research performance. London: Performance Press, 2014. (Wenner-Gren International Series, v.87). Available from: <http://www. portlandpress.com/pp/books/online/wg87/087/0107/ 0870107.pdf>. Cited: Sept. 15, 2014. 
